\title{
Simulating electro-coating of automotive body parts using BEM
}

\author{
J. M. W. Baynham ${ }^{1}$, R. Adey ${ }^{1}$, V. Murugaian ${ }^{2}$ \& D. Williams ${ }^{2}$ \\ ${ }^{1} C M$ BEASY Ltd, Ashurst Lodge, Southampton, UK \\ ${ }^{2}$ IARC (The International Automotive Research Centre), \\ Warwick Manufacturing Group, The University of Warwick, \\ Coventry, UK
}

\begin{abstract}
The simulation of electro painting has rapidly become a "must-have" tool for auto-manufacturers over the past three to four years. Various approaches have been used in such simulation, including both FE and BE.

In this paper details of the boundary element based "BEASY" Electro-coat Modelling software are presented which allow car-body parts to be simulated. Examples are presented showing application of the method. Drawbacks of the method are discussed.
\end{abstract}

\section{Introduction}

One electro-painting process in use today involves immersing a metallic (conducting) structure in a tank of water based paint, then passing a direct current from anodes (also immersed in the paint) to the metallic structure which forms the cathode.

Electrochemical processes at the cathode surface cause deposition of paint on the surface. Since the deposited paint has electrical resistance, the electrical fields in the tank are constantly changing as more and more paint is deposited.

Paint is deposited most rapidly where the current density is greatest. The metal surfaces inside any cavities initially receive no current, but as the paint film builds up on exterior surfaces, current begins to penetrate the cavities, and deposition occurs here too.

The difficulty of coating inside cavities is that the wet paint film is never a perfect insulator. Consequently current passing along the axis inside a hollow 
tubular cathode (for example) reduces steadily along the axis as current flows sideways into the walls.

The challenge for auto designers is to ensure that adequate paint film thickness is achieved inside such cavities, because film thickness directly affects corrosion resistance.

The incentive to use simulation is strong, since it allows design verification at an early stage of the overall design process. The costs of design changes early in the design process are significantly lower than later on. However the most significant advantage that simulation confers is compression of the Product Development process. The associated reductions in cost improve operating performance, and time savings enable manufacturers to shorten time to market and improve responsiveness to consumer requirements.

Simulation of electro-painting requires first a way to determine the currents flowing in the electrolyte (the paint). The boundary element method (BEM) has been applied to the very similar application of corrosion simulation since the late 1970s [1]. In both electro-painting and corrosion simulation the domain is the electrolyte surrounding the structure, and BEM is well suited to such situations because creating meshes on the surfaces is relatively straightforward.

Secondly, the simulation must provide a way to represent the paint film layer. From the electrical point of view the important property of the paint film is simply its resistance.

Finally, the simulation must somehow represent the build-up of the paint film thickness. In transient simulation this implies a time-stepping algorithm which uses current density to determine rate of deposition, and then incrementally increases the recorded film thickness.

\section{Methodology}

\subsection{The BEM solver}

The ability to solve the Laplacian using BEM is well documented, see for example [2]. The BEM is a mixed method, which means that both voltage on the surface (of the electrolyte) and current density in the normal direction through the surface are represented directly.

By contrast in the FEM domain method where only voltage is represented directly, the current density is obtained by differentiating the voltage field. For reasons of accuracy the differentiation is normally performed at positions inside the volume element (away from the sides) and the result is extrapolated to the surface.

The current densities produced by the mixed method BEM are therefore generally more accurate than those produced by the FEM. This means the BEM elements can be bigger than those used in a corresponding FEM model

In $3 \mathrm{D}$ the boundary elements are a mixture of triangular and quadrilateral shapes, each representing a part of the surface of the domain. In the BEM each degree of freedom is linked mathematically with every other degree of freedom. 
For sheet metal cathodes, each side of the metal sheet is a surface of the electrolyte, and hence must have elements on it. This can cause problems when the metal sheet is thin, because the collocation, direct method BEM equations for a pair of nodes separated by the sheet thickness become more and more similar as the thickness reduces. For a "zero thickness" sheet the equations would be identical, so the matrices are singular and cannot be solved. For small but nonzero thickness the equations will be ill-conditioned, leading to problems when performing equation solution, and possible inaccuracy.

One approach to overcome this problem is to divide the electrolyte into multiple domains (or zones) so that opposite sides of the metal sheet are in different zones. This method works very well, but can lead to complication in model-building because artificial boundaries must be constructed inside the domain. An example of application of this method is shown in Figure 1 which shows a model of a "throw-box" (three metal sheets held some millimetres apart to form two cavities). The figure shows the boundaries of three zones, and how they fit together to form the complete model. It also shows the artificial boundary which covers the access from outside into cavity 1 . In the model shown creating the artificial boundary was not difficult, but in a complex car part, the task is much harder.

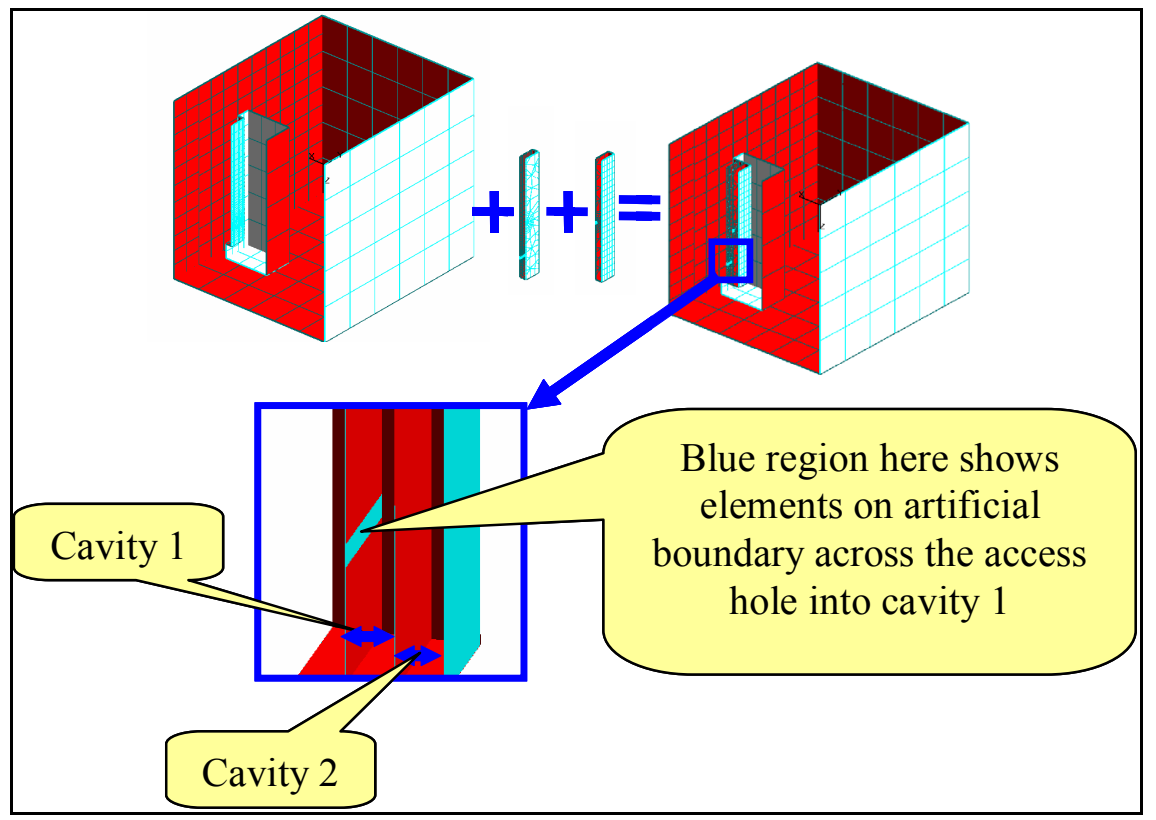

Figure 1: $\quad$ Multiple zone model of throw-box with two cavities.

If the metal sheet is approximated as having zero thickness, a different mathematical approach can be used when generating the equation for the node on the "other" side of the sheet. Consequently the equations for the pair of nodes are 
different, and the matrix is no longer singular. This technique, known in BEM as dual elements [3], is advantageous for the user since it is only necessary to create one element using a pre-processor. The dual can be generated automatically inside the BEM solver. An example of application of this method is shown in Figure 2, which again shows the throw-box model. Note the absence of elements across the entrance to the cavity.

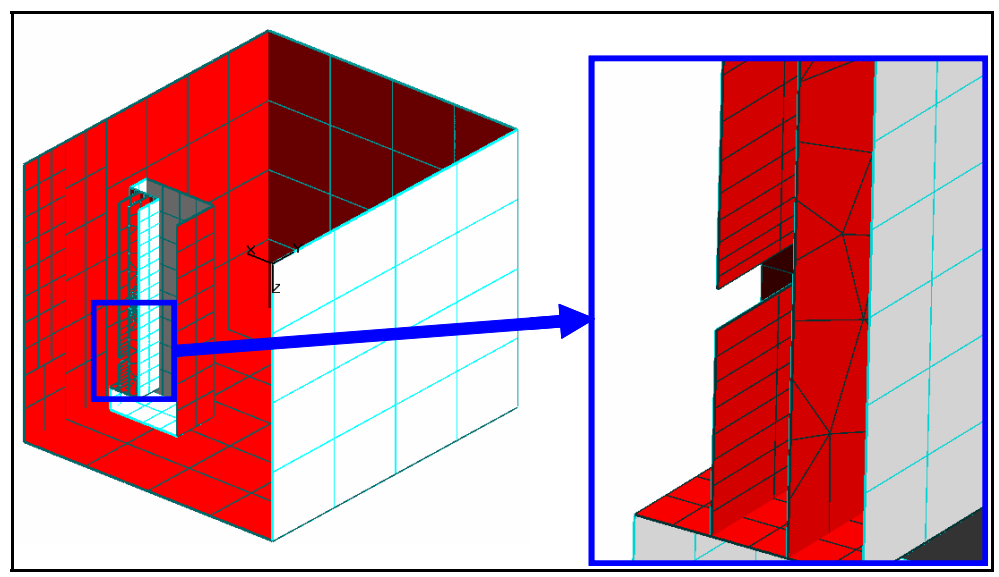

Figure 2: $\quad$ Single zone model using dual elements.

\subsection{The paint film}

The paint film can be represented very simply as a resistive boundary condition relating the current density flowing from the electrolyte into the metal, the voltage in the metal, and the voltage in the electrolyte just above the paint film by and equation of the form:

$$
\mathrm{j}_{\mathrm{n}} \cdot \mathrm{R}_{\text {film }}=\mathrm{V}_{\text {electrolyte }}-\mathrm{V}_{\text {metal }}
$$

This treatment does not take into account any geometrical change of shape of the electrolyte domain (not a problem since in practise the thickness of the $d r y$ paint film is not likely to be more than 30 microns). It is possible however that deposition may block any small gaps between metal sheets.

\subsection{The deposition process}

The rate of deposition of paint may be expressed as:

where:

$$
\mathrm{dh} / \mathrm{dt}=\mathrm{C}_{\mathrm{eff}}\left(\mathrm{j}-\mathrm{j}_{0}\right)
$$

$\mathrm{dh} / \mathrm{dt}$ is the rate of change of the (dry) film thickness

$\mathrm{C}_{\text {eff }}$ is the Coulombic efficiency of the paint, expressed as a volume of paint per Coulomb of charge

$\mathrm{j}$ is the current density flowing from the electrolyte into the metal $\mathrm{j}_{0}$ is the current density below which no deposition occurs. 


\subsection{The relation between thickness and resistance}

The relationship between film thickness and film resistance can be expressed in tabular form. This allows non-linear variation, and makes it possible to account for the resistance of any pre-treatments.

\section{The simulation process}

For transient simulation the process is as follows:

- create influence matrices;

- apply cathode voltage to the sheet metal;

- $\quad$ solve for current density;

- integrate through time to obtain the change of film thickness;

- where film thickness is non-zero apply a resistive boundary condition;

- again solve for current density;

- repeat for the required duration of the simulation.

Clearly the magnitude of the time step must be controlled, since too big a step is likely to result in unrealistic distribution of film thickness. In practise it is found better to choose a delta(h) (i.e. thickness change) which is used to limit the time step.

For the direct method, the simulation process is similar, except that instead of integrating through time, the boundary condition applied to the cathode is switched between cathode voltage and target deposition current density.

Both methods provide the film thickness at the target time.

\section{Application}

\subsection{Car door}

The direct method of simulation has been applied to the car-door model shown in Figure 3.

The door was meshed at the IARC using Hypermesh, and exported as a NASTRAN bulk data file containing shell elements. This file was processed into a BEASY data file containing dual elements, and elements representing the paint bath sides and base, and the paint surface were added. Typically anode geometry would also be constructed, but in this case voltage boundary conditions were simply applied to some parts of the tank sides. Similarly the paint free surface is usually treated as a symmetry plane, so no elements are required on it. The total anode area was $21600 \mathrm{~cm}^{2}$, and the anodes were assumed to be at 340 volts. The total cathode area was $43,540 \mathrm{~cm}^{2}$.

The direct method was applied, using assumed paint properties, and wet film resistance $85 \mathrm{MegaOhm} . \mathrm{mm} 2$ at film thickness corresponding to a 25 micron dry film thickness.

The paint film thickness at target time 180 seconds is shown in Figure 4 to Figure 8 . 


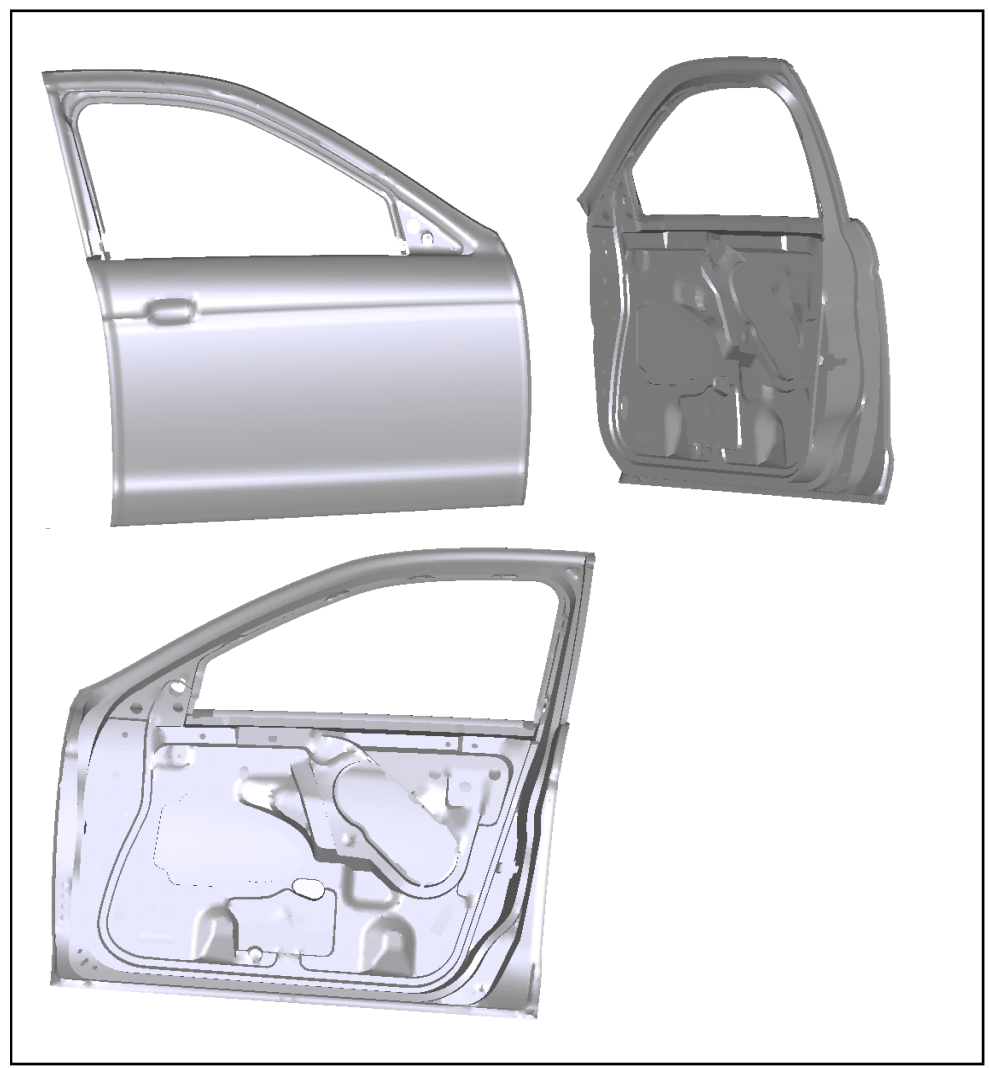

Figure 3: Door used in simulation.

\subsection{Discussion of results}

The film build on the outer surfaces on the "outer side" of the car door takes the expected form (Figure 4), that is: nearly constant thickness except near access holes into the interior of the door cavities.

Figure 5 shows paint thickness on the reverse of the metal sheets, but again viewed from the outside of the car. This figure shows a gradual reduction of thickness along the "axis" of the cavities, shown arrowed red in the figure. This gradual reduction is consistent with results of throw-tube experiments.

Figure 6 shows paint thickness as viewed from the inside of the car.

Figure 7 shows a detail of film build (using a different contour plot scale) on the reverse side of the metal sheets near the top left of the door when viewed from the outside of the car. The red arrows in the figure highlight regions where the film build is bigger than it is to either side along the axis of the cavity. The only way this can happen is if current is able to flow into the cavity nearby, and by looking at the same region from the inside of the car (Figure 8) the reason can 
be seen. These small holes (shown by red arrows on the right hand part of Figure 8) allow enough current to flow into the cavity to prevent the paint thickness from reducing too far.

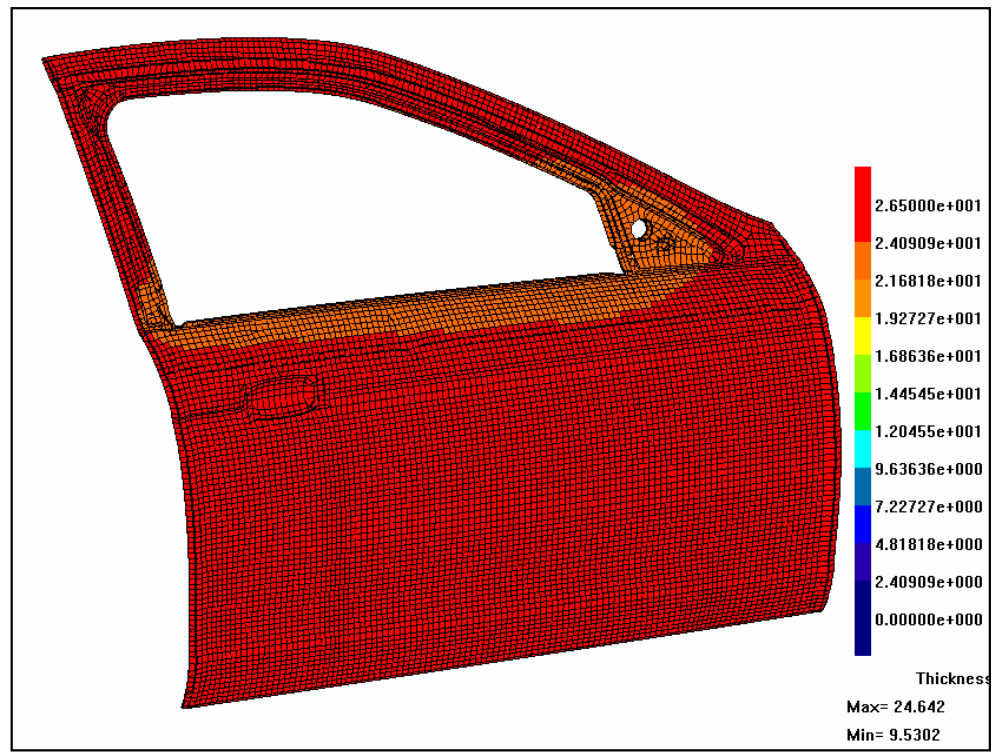

Figure 4: Distribution of film thickness, viewed from "outside" the car.

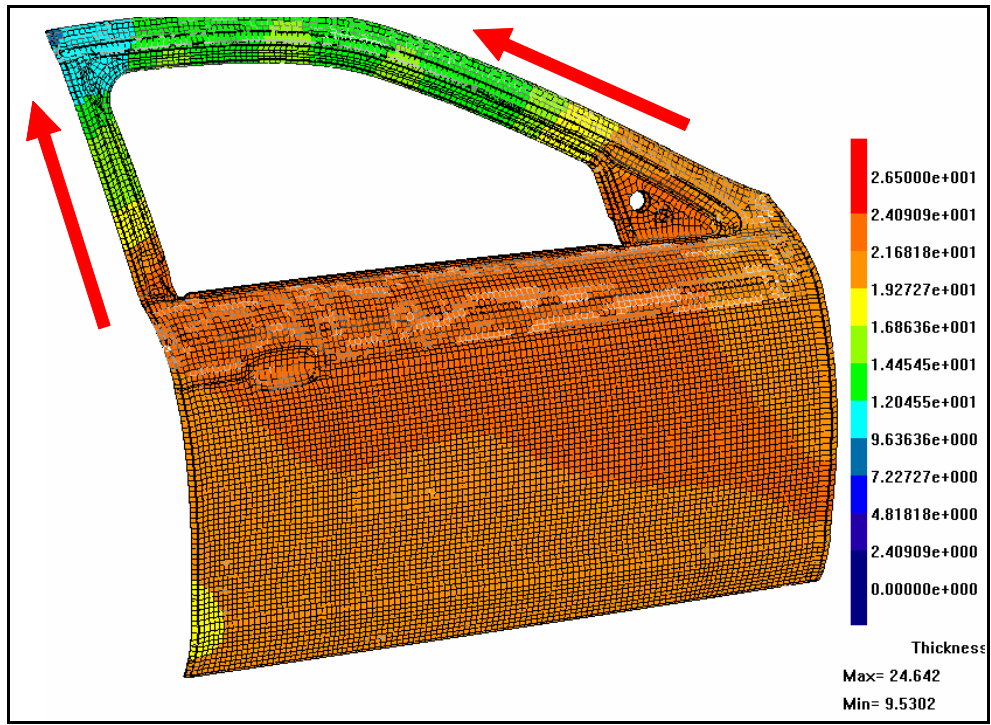

Figure 5: Distribution of film thickness on the "reverse side" of the metal sheets, viewed from "outside" the car. 


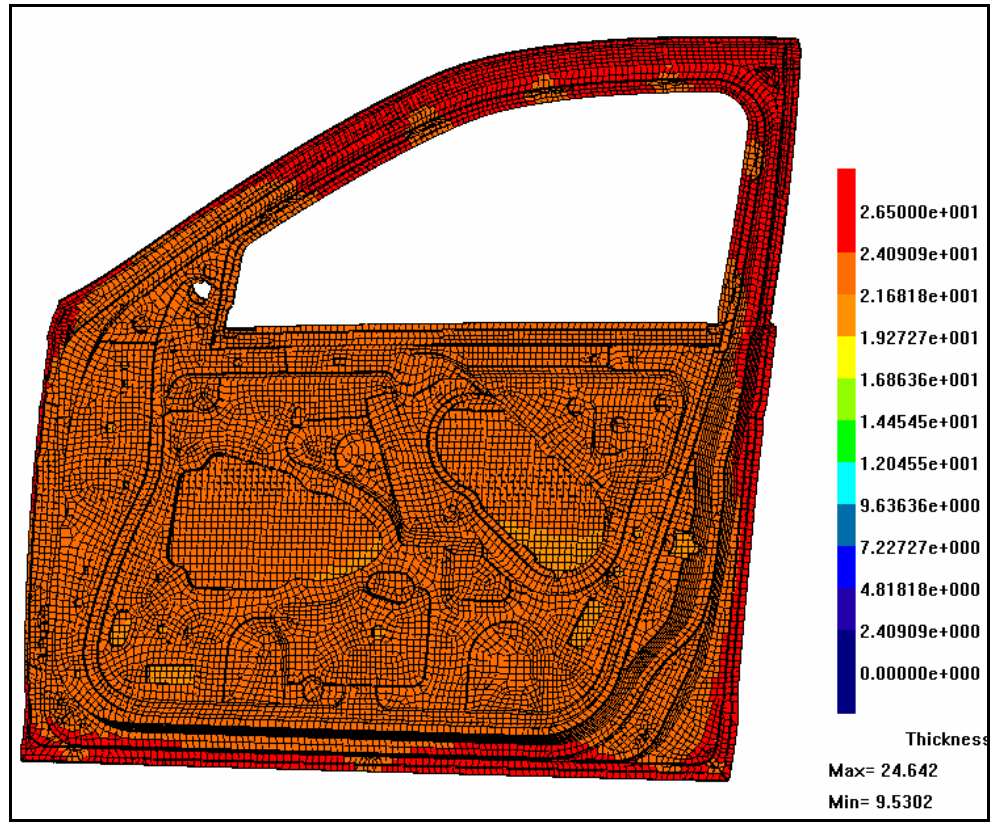

Figure 6: Film thickness, viewed from "inside the car".

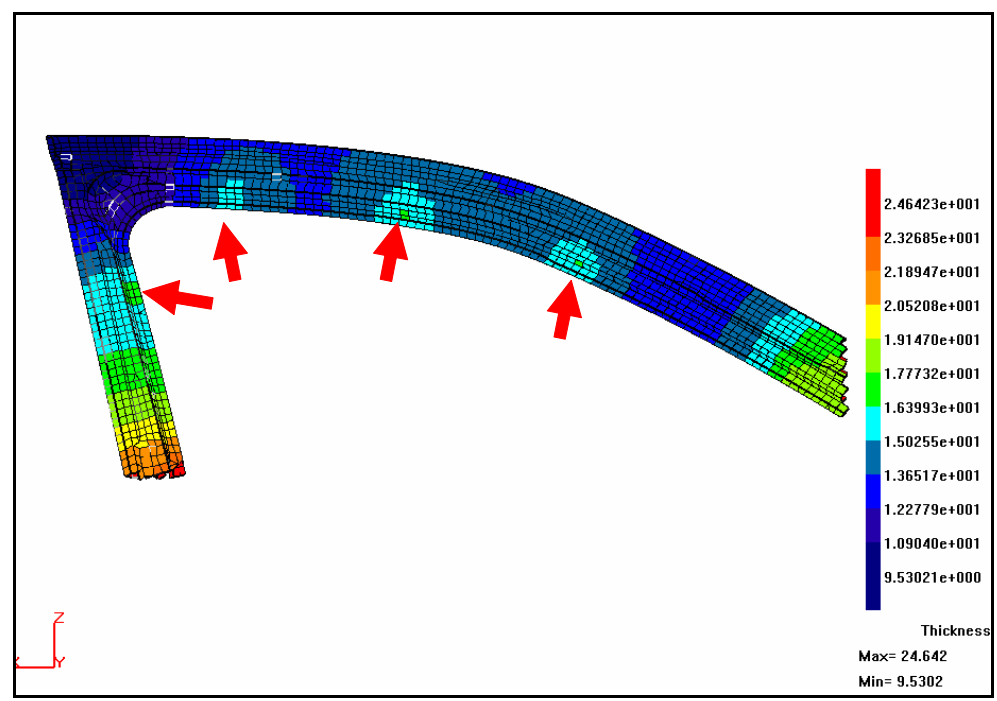

Figure 7: Distribution of film thickness on inner surfaces of the cavity, viewed from "outside" the car. 


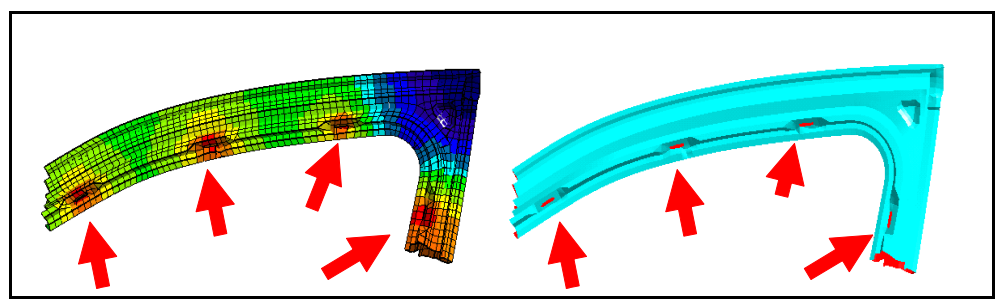

Figure 8: Distribution of film thickness on inner surfaces of the cavity, viewed from "inside" the car, and corresponding view of geometry.

\subsection{Comparison with Experimental Measurements}

Experimental measurements were made by the IARC and compared with simulation results from a model similar to, but less refined than that shown in the previous section. The comparison, shown in Figure 9, suggests good correlation between simulation and experiment.

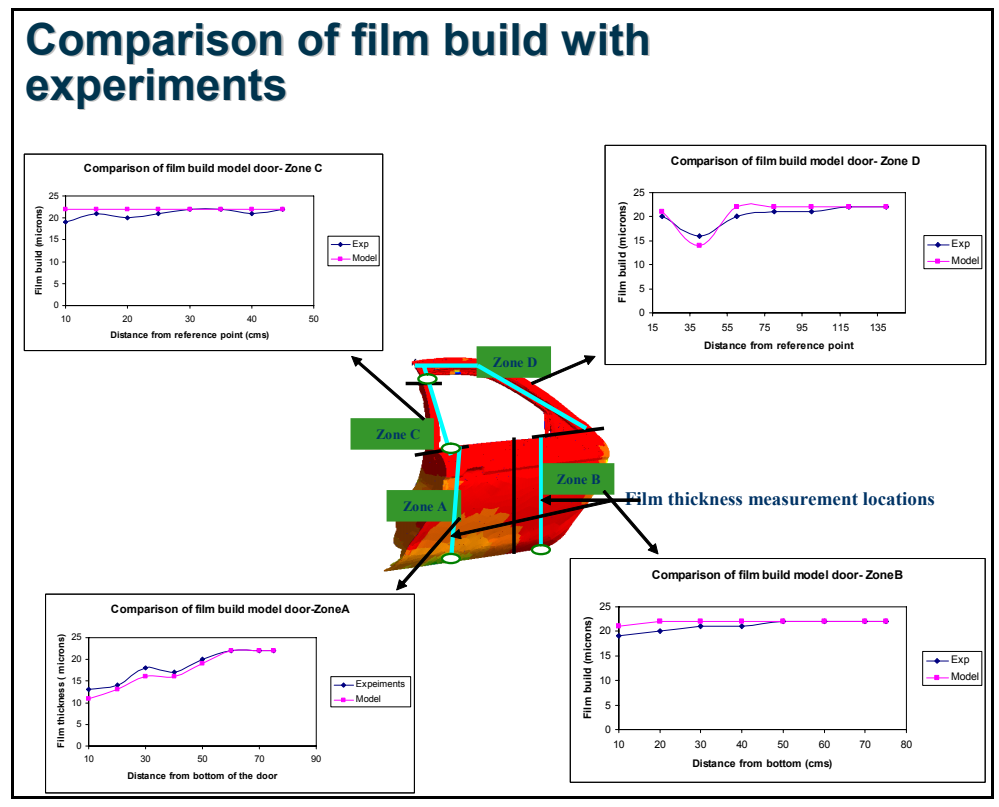

Figure 9: $\quad$ Showing comparison between simulation and experimental results.

\section{Design changes}

The method described using BEM is very suitable for simulation of design changes, because modification to add an extra paint access hole requires minimal change to the mesh. The change can be effected at a local element level, with no 
other changes required to the model. For example Figure 10 shows the mesh for a model before and after a "design change". The only change to the mesh is in the area shown circled.

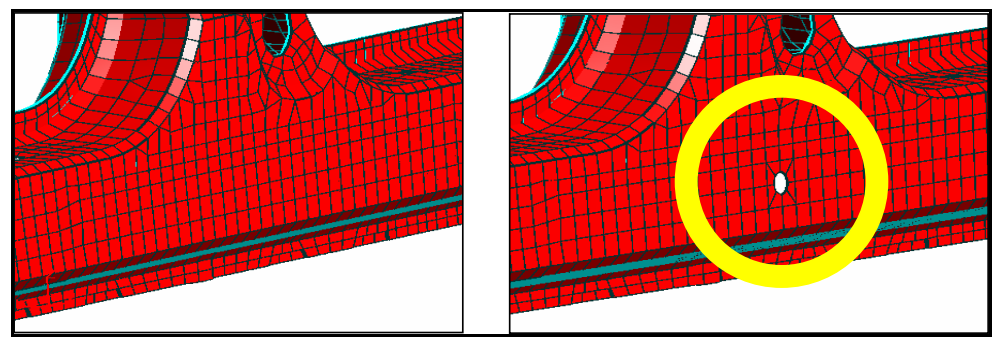

Figure 10: Mesh changes required to add a new paint access hole.

\section{Drawbacks of the method}

Although the idea of using a shell mesh of a car part as the mesh for BEM electro-coating simulation is attractive (in particular since such meshes must be created for structural simulation), there are some caveats as follows.

Firstly, the dual elements have zero thickness. This creates a problem if the elements are created on the neutral axis of the metal sheets, since there will be a gap between the dual elements where the metal sheets are joined together.

Secondly, at joints the metal sheets overlap slightly, and there is no electrolyte between the sheets. However the dual elements do not represent this situation since they will be separated by the thickness of the metal sheet.

These issues mean that in practise the geometry used to create the dual elements must be modified near joints, to remove overlaps and gaps. Figure 11 shows an example of how this geometry modification might be done.

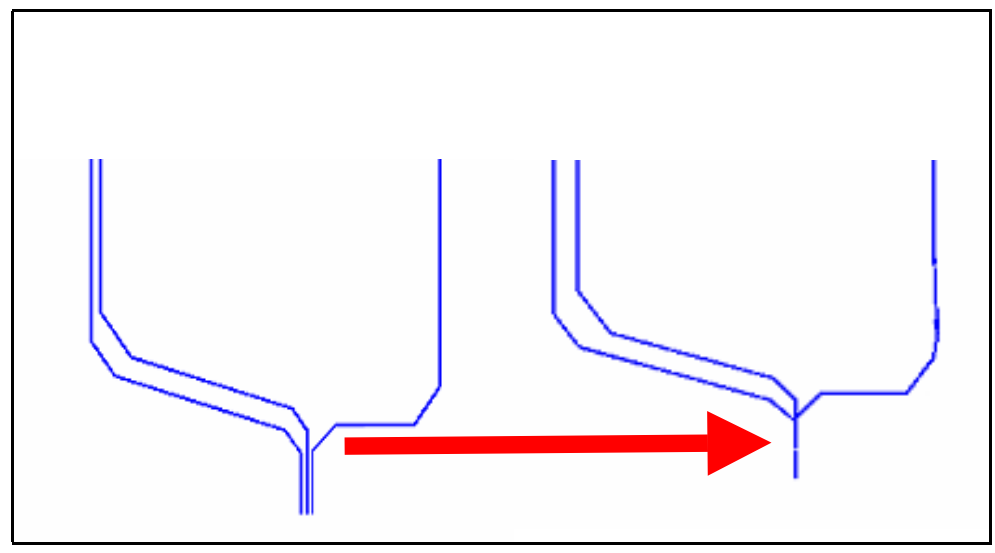

Figure 11: Geometry change to remove overlaps and gaps at joints. 


\section{Conclusions}

The successful application of BEM technology to simulation of electro-coating of automotive parts has been demonstrated.

A notable feature of the methodology is the ease with which design changes can be incorporated for evaluation and the attendant virtual engineering performance benefits.

\section{Acknowledgement}

The authors are grateful to Jaguar and Land Rover for permission to show the door model.

\section{References}

[1] Danson, D.J. and Warne, M.A. Current Density/Voltage Calculations Using Boundary Element Techniques, Corrosion/83. 1983.

[2] Brebbia, C.A. The Boundary Element Method for Engineers, Pentech Press. London, 1978.

[3] Hong, H. and Chen, J. Derivations of integral equations in elasticity. Journal of Engineering Mechanics, 114(6), pp1028-1044, 1988. 